\title{
Preoperative neutrophil-lymphocyte ratio and fibrinogen level in patients distinguish between muscle-invasive bladder cancer and non-muscle- invasive bladder cancer
}

\author{
This article was published in the following Dove Press journal: \\ OncoTargets and Therapy \\ 8 August 2016 \\ Number of times this article has been viewed
}

\author{
Chengquan $\mathrm{Ma}{ }^{1}$ \\ Bingxin $\mathrm{Lu}^{2}$ \\ Chengwen Diao² \\ Kun Zhao ${ }^{2}$ \\ Xinpeng Wang ${ }^{2}$ \\ Baojing $\mathrm{Ma}^{2}$ \\ Baojian Lu \\ Erlin Sun ${ }^{2}$ \\ 'Tianjin Institute of Urology, The \\ Second Hospital of Tianjin Medical \\ University, Tianjin, People's Republic \\ of China; ${ }^{2}$ Tianjin Key Lab of Urology \\ Basic Science, Tianjin Institute of \\ Urology, The Second Hospital of \\ Tianjin Medical University, Tianjin, \\ People's Republic of China
}

Introduction: The aim of this study was to explore if the preoperative neutrophil-lymphocyte ratio (NLR) and fibrinogen level can help in distinguishing between muscle-invasive bladder cancer (MIBC) and non-muscle-invasive bladder cancer (NMIBC).

Methods: We identified 669 patients who underwent surgery at our institution, and evaluated their preoperative NLRs and fibrinogen levels. Patients were divided into two groups, NMIBC (group-I) and MIBC (group-II), according to the postoperative pathology. For the intergroup comparison, data obtained from the two groups were evaluated using independent samples $t$-test. The cutoff value of the NLR, fibrinogen level, and integrated NLR and fibrinogen level was determined with receiver operating characteristic (ROC) curve.

Results: The mean NLRs of group-I and group-II were found as 2.71 \pm 2.46 and 4.66 \pm 8.00 , respectively $(P<0.001)$. The fibrinogen levels of the two groups were $\sim 3.13 \pm 0.70 \mathrm{~g} / \mathrm{L}$ and $3.41 \pm 0.84 \mathrm{~g} / \mathrm{L}$, respectively $(P=0.001)$. Whether the NLR, fibrinogen level, and integrated NLR and fibrinogen level can help in distinguishing between MIBC and NMIBC was evaluated with ROC curve. The cutoff value of NLR was estimated as 2.01 according to the Youden index. With this value, sensitivity was found as $67.1 \%$, specificity was $52.7 \%$, and area under receiver operating characteristic (ROC) curve (AUC) was $0.601(P=0.031)$. The cutoff value of fibrinogen level was estimated as $3.17 \mathrm{~g} / \mathrm{L}$ according to the Youden index. Accordingly, sensitivity was found as $58 \%$, specificity was $58 \%$, and AUC was $0.60(P=0.001)$. The cutoff value of integrated NLR and fibrinogen level was found as 0.166 ; the sensitivity was found as $86 \%$, specificity was $42 \%$, and AUC was $0.801(P=0.01)$.

Conclusion: The data obtained in this study suggested that $67.1 \%$ of Ta-T1 tumors were likely to be invasive if the NLR was $>2.01$ and $58 \%$ were likely to be invasive if the fibrinogen level was $>3.17 \mathrm{~g} / \mathrm{L}$. When we used both the NLR and fibrinogen level to distinguish between the MIBC and NMIBC, sensitivity was found to be $86 \%$, and specificity was $42 \%$.

Keywords: NLR, fibrinogen, NMIBC, MIBC, ROC

\section{Introduction}

According to statistics, bladder carcinoma is the seventh most common malignancy globally. Approximately 430,000 new cases are diagnosed annually, and <165,000 cancer-related deaths occur each year. ${ }^{1}$ Approximately $25 \%$ of the bladder cancers (BCs) are found to be muscle invasive (MIBC) nowadays. Patients with MIBC are at high risk of cancer-related death even after undergoing active treatment, with a 5-year survival proportion of $33 \%$ and $5.4 \%$ for local and distant metastatic MIBC, respectively. ${ }^{2}$
Tianjin Key Lab of Urology Basic Science, Tianjin Institute of Urology, The Second Hospital of Tianjin Medical University, Tianjin 3002II, People's Republic of China

Tel $+86-13032259605$

Fax +86-2I-64085785

Email mchengquan@yahoo.com 
At present, cystoscopy and voided urine cytology remain the basic standard of $\mathrm{BC}$ diagnosis. ${ }^{3}$ Although urine cytology has a high sensitivity in the detection of highgrade $\mathrm{BC}$, it lacks sensitivity (ranging from $4 \%$ to $31 \%$ ) to diagnose low-grade tumors. Cystoscopy is an invasive, expensive, painful, and potentially infectious procedure. ${ }^{4}$ The development of new tools for the early diagnosis and discrimination of non-muscle-invasive BCs (NMIBCs) and MIBCs is a challenge in the field of uro-oncology, and it could provide benefit of invasiveness and help to take positive measures to reduce harm from malignant BCs. In addition, the accuracy of pathological diagnosis which was reduced by the limitation of specimen collection needs to be improved. ${ }^{5}$

Despite standard treatments for MIBC such as radical cystectomy (RC) and pelvic node dissection, 5-year overall survival rates have been shown to be between $26 \%$ and $77 \% .{ }^{6}$ One study revealed that trimodal treatment seems to lead to a better outcome in patients with MIBC compared with $\mathrm{RC}$, and the median 5-year overall survival rate was $57 \%$ in the trimodal treatment group, when compared with $52 \%, 51 \%$, and $53 \%$ in the whole group receiving $\mathrm{RC}$ or the group treated with RC alone or RC + chemotherapy, respectively. ${ }^{7}$ Thus, an efficient early diagnostic approach is crucial to improve survival of BC patients. Many urothelial cell carcinoma (UCC) indicators such as microsatellite analysis, ImmunoCyt, NMP22, CYFRA21-1, Lewis-X, FISH, and hemoglobin $(\mathrm{Hb})$ dipstick molecular tests and urine markers have been examined; however, due to the low sensitivity and specificity, they did not become a standard procedure for prognosis and diagnosis of patients with UCC. ${ }^{8,9}$ To date, many studies have revealed that neutrophil-lymphocyte ratio (NLR) would be associated with multiple types of cancers such as lung, breast, prostate, glioblastoma, colon, bladder, kidney, and pancreatic cancers. ${ }^{10-17}$ Different types of studies have revealed that NLR could possibly be valuable in timely detection, informing prognoses, and monitoring treatment effects.

In addition, a number of studies suggest that an interactive relationship exists between hemostatic factors and tumor biology. ${ }^{18-21}$ A number of procoagulant and fibrinolytic factors have been discovered to be overexpressed in tumors. One of these factors is plasma fibrinogen. Recent studies have revealed that elevated preoperative plasma fibrinogen levels are associated with worse outcome in various malignancies; however, the detecting value of plasma fibrinogen levels for bladder carcinoma has not yet been reported. In this study, we aimed to research if the integrated NLR and fibrinogen level has significance in discriminating between MIBC and NMIBC.

\section{Methods}

We retrospectively evaluated the data of 669 patients with $\mathrm{BC}$ who underwent surgery and were monitored at the Second Hospital of Tianjin Medical University from 2010 to 2015. This study was approved by the Ethics Committee of The Second Hospital of Tianjin Medical University. All the patients signed an informed consent form to be included in this study. The tumors were graded according to the World Health Organization-International Society of Urologic Pathology 2004 guidelines and the TNM 2002 staging system. All of the pertinent laboratory and pathology results (tumor size and number, presence of carcinoma in situ, transurethral resection [TUR] and reoperation, postoperative immunotherapy, and chemotherapy), and medical data were obtained from the hospital databases.

Data obtained from the routine preoperative test results of the patients included the neutrophil and lymphocyte counts, NLR, levels of fibrinogen, $\mathrm{Hb}$, red blood cells (RBCs), mean corpuscular $\mathrm{Hb}$, mean corpuscular $\mathrm{Hb}$ concentration, creatinine, glucose, albumin total protein, and monocytes, and age. The NLR was determined by dividing the absolute neutrophil count by the absolute lymphocyte count. Patients were divided into two groups, NMIBC (group-I) and MIBC (group-II), according to their pathologies after the primary TUR and reoperation (including secondary TUR, RC, and partial resection of the bladder).

Statistical analyses were performed using IBM SPSS Statistics for Windows, version 20.0 (IBM Corporation, Armonk, NY, USA). For intergroup comparison, data obtained from NMIBC and MIBC groups were evaluated using independent samples $t$-test. The cutoff value of the NLR, fibrinogen level, and integrated NLR and fibrinogen level was determined with receiver operating characteristic (ROC) curve. $P<0.05$ was accepted as statistically significant, and $P<0.01$ as extremely significantly different.

\section{Results}

According to the study results, group-I consisted of 579 patients, while group-II consisted of 90 patients. Mean age of patients in group-I and group-II was $65.21 \pm 11.9$ and $67.46 \pm 11.4$ years, respectively. The NLRs of group-I and group-II were found as $2.71 \pm 2.46$ and $4.66 \pm 8.00$, respectively $(P<0.001)$. The fibrinogen levels of group-I and group-II were found as $3.13 \pm 0.70$ and $3.41 \pm 0.84 \mathrm{~g} / \mathrm{L}$, respectively $(P=0.001)$. Data of group-I and group-II 
Table I The demographics, risk factors, and clinicopathological characteristics of the two groups of patients

\begin{tabular}{|c|c|c|c|}
\hline Parameters & $\begin{array}{l}\text { NMIBC (579), } \\
\text { mean } \pm \text { SD }\end{array}$ & $\begin{array}{l}\text { MIBC }(90) \\
\text { mean } \pm \text { SD }\end{array}$ & $P$-value \\
\hline Age (years) & $65.20 \pm 11.95$ & $67 \pm 11.40$ & 0.093 \\
\hline WBCs $\left(10^{9} / \mathrm{L}\right)$ & $6.63 \pm 2.24$ & $8.11 \pm 7.72$ & 0.000 \\
\hline Neutrophils $\left(10^{9} / \mathrm{L}\right)$ & $4.21 \pm 2.24$ & $5.76 \pm 7.68$ & 0.000 \\
\hline Lymphocytes $\left(10^{9} / \mathrm{L}\right)$ & $2.12 \pm 6.38$ & $\mathrm{I} .72 \pm 0.72$ & 0.553 \\
\hline NLR & $2.7 I \pm 2.46$ & $4.66 \pm 8.00$ & 0.00 \\
\hline Monocytes $\left(10^{8} / \mathrm{L}\right)$ & $6.15 \pm 1.75$ & $5.74 \pm 2.02$ & 0.047 \\
\hline $\operatorname{RBCs}\left(10^{12} \mathrm{~g} / \mathrm{L}\right)$ & $4.5 \mathrm{I} \pm 0.54$ & $4.27 \pm 0.62$ & 0.00 \\
\hline $\mathrm{Hb}(\mathrm{g} / \mathrm{L})$ & $140.49 \pm 18.06$ & $132.45 \pm 20.25$ & 0.000 \\
\hline $\mathrm{MCH}(\mathrm{pg})$ & $31.28 \pm 2.05$ & $30.98 \pm 1.77$ & 0.194 \\
\hline $\mathrm{MCHC}(\mathrm{g} / \mathrm{L})$ & $339.09 \pm 8.78$ & $338.20 \pm 8.79$ & 0.368 \\
\hline Creatinine $(\mu \mathrm{mol} / \mathrm{L})$ & $80.16 \pm 40.76$ & $80.06 \pm 29.80$ & 0.983 \\
\hline Glucose (mmol/L) & $5.62 \pm 1.57$ & $5.93 \pm 1.73$ & 0.105 \\
\hline Fibrinogen (g/L) & $3.13 \pm 0.70$ & $3.4 I \pm 0.84$ & 0.001 \\
\hline Albumin (g/L) & $41.60 \pm 5.22$ & $39.97 \pm 5.69$ & 0.008 \\
\hline Total protein $(\mathrm{g} / \mathrm{L})$ & $66.07 \pm 6.53$ & $65.44 \pm 6.84$ & 0.412 \\
\hline
\end{tabular}

Note: For intergroup comparison, data obtained from NMIBC and MIBC groups were evaluated using independent samples $t$-test.

Abbreviations: NMIBC, non-muscle-invasive bladder cancer; SD, standard deviation; MIBC, muscle-invasive bladder cancer; WBCs, white blood cells; NLR, neutrophil-lymphocyte ratio; RBCs, red blood cells; Hb, hemoglobin; $\mathrm{MCH}$, mean corpuscular $\mathrm{Hb}$; $\mathrm{MCHC}$, mean corpuscular $\mathrm{Hb}$ concentration.

are summarized in Table 1 (including the neutrophil and lymphocyte counts, NLR, levels of fibrinogen, $\mathrm{Hb}, \mathrm{RBCs}$, $\mathrm{MCH}, \mathrm{MCHC}$, creatinine, glucose, albumin total protein, and monocytes, and age). From Table 1, we can understand that mean of white blood cells, neutrophils, NLR, and fibrinogen was significantly higher in MIBC $(P<0.05)$, while that of monocytes, $\mathrm{RBCs}, \mathrm{Hb}$, and albumin was significantly higher in NMIBC $(P<0.05)$. Whether the NLR or fibrinogen level can distinguish between tumors in group-I and group-II was evaluated with ROC curve; the cutoff value was found as 2.01 for NLR and $3.17 \mathrm{~g} / \mathrm{L}$ for fibrinogen level according to the Youden index (Figure 1). For these values, sensitivity was determined as $67.1 \%$ and $58 \%$, specificity as $52.7 \%$ and $58 \%$, and area under the concentration-time curve (AUC) as $0.601(P=0.031$, confidence interval $=0.547-0.653)$ and 0.60 $(P=0.001$, confidence interval $=0.551-0.651)$, respectively. The cutoff value of integrated NLR and fibrinogen level was found as 0.166 ; hereby, the sensitivity was $86 \%$, specificity was $42 \%$, and AUC was 0.801 ( $P=0.01$, confidence interval $=0.763-0.838$ ).

\section{Discussion}

The development of new tools for the early diagnosis and prognosis of $\mathrm{BC}$ is a challenge in the field of uro-oncology. It is therefore feasible in clinical practice to assess malignancy of cancer with simple, inexpensive, and objective



Figure I ROC curves for NLR, Fibrinogen and NLR + fibrinogen.

Notes: According to the cutoff value of NLR, sensitivity was found as $67.1 \%$, specificity was $52.7 \%$, and AUC was 0.601 ( $P=0.031$, confidence interval $=0.547$ 0.653 ). According to the cutoff value of fibrinogen, sensitivity was found as $58 \%$, specificity was $58 \%$, and $A \cup C$ was 0.60 ( $P=0.00 \mathrm{I}$, confidence interval $=0.55 \mathrm{I}-0.65 \mathrm{I}$ ). According to the cutoff value of integrated NLR and fibrinogen level, sensitivity was found as $86 \%$, specificity was $42 \%$, and AUC was 0.801 ( $P=0.01$, confidence interval $=0.763-0.838$ ).

Abbreviations: NLR, neutrophil-lymphocyte ratio; AUC, area under the ROC curve; ROC, receiver operating characteristic.

tools. The aim of our study was to assess whether the NLR or fibrinogen level would be useful in predicting disease severity in BC patients. Our finding may help urologists in counseling patients about their risk of upstaged disease and make an adminicular decision on whether to perform early cystectomy or neoadjuvant treatment. Raj et al proved that neoadjuvant chemotherapy improved survival times of $\mathrm{T} 2$ $\mathrm{BC}$ patients who underwent RC. This benefit was more obviously seen in those with extravesical, advanced malignant neoplasms. ${ }^{22}$

Inflammatory and immune cells are the basic constituents of the tumor microenvironment. Inflammation might frequently be associated with different stages of tumor development, including initiation, promotion, malignant conversion, invasion, and metastasis. ${ }^{23}$ Several articles have revealed the prognostic value of various inflammatory factors such as albumin, C-reactive protein, Glasgow prognostic score, and prognostic index in cancer patients. The NLR represents an easily measured, reproducible, and inexpensive marker of systemic inflammation which has been confirmed to be associated with oncologic outcomes in multiple malignancies, including breast, colorectal, lung, and gastric cancers. Gondo et al stated that NLR threshold 
value $<2.5$ and $\geq 2.5$ was an independent prognostic factor; additionally, tumor size, hydronephrosis, $\mathrm{Hb}$ levels, and the combination of these factors could stratify disease-specific survival risks in $\mathrm{BC}$ patients undergoing RC. ${ }^{24}$ Azuma et al revealed that preoperative NLR was intensively related to a poor prognosis in patients with upper urinary tract UCC as an indication of pathologic $\mathrm{T}$ stage and tumor grade. In their study, it was shown that NLR $\geq 2.5$ could predict local tumor aggressiveness and independently forecast the local pathologic situation, and NLR was obviously associated with recurrence-free survival and cancer-specific survival rates. ${ }^{25}$ Ozyalvacli et al reported that the best cutoff value of the NLR as an indicator of recurrence and progression in BC patients was $\geq 2.43$; patients with an NLR above this value experienced significantly higher recurrence without progression, highlighting the potential role of the NLR in pT1 superficial bladder tumors. ${ }^{26}$ Moreover, Ceylan et al reported that the cutoff value was found as 3.96 through the Youden index; for this value, sensitivity was calculated as $50 \%$, specificity as $22.8 \%$, and AUC as $0.615 .{ }^{27}$ Reportedly, patients who were upstaged to $\geq \mathrm{pT} 3$ demonstrated statistically greater NLRs compared with patients who stayed at $\leq$ pT2 stage.$^{28}$ Other studies have revealed that this result may be useful in prognosis for BC patients. ${ }^{15,29-33}$

Fibrinogen is synthesized in the liver as a $350 \mathrm{kDa}$ glycoprotein and converted to insoluble fibrin by activated thrombin. Fibrinogen plays a part in blood coagulation, thrombosis, wound healing, and platelet aggregation. Recently, much attention was attracted to the relationship between hypercoagulation and the progression of malignancies. Moreover, recent studies suggest that fibrinogen is associated with tumor evolution including ovarian, ${ }^{34}$ breast, ${ }^{35}$ non-metastatic renal cell, ${ }^{36}$ cervical, ${ }^{37}$ endometrial, ${ }^{38}$ esophageal, ${ }^{39}$ colon, ${ }^{40}$ prostate,${ }^{41}$ endometrial, ${ }^{42}$ non-small-cell lung, ${ }^{43}$ and rectal cancers. ${ }^{44}$ According to our knowledge, this is the only study evaluating the relationship between $\mathrm{BC}$ and fibrinogen in literature. Also, the fibrinogen was unknown to be an independent prognostic factor in predicting survival.

ROC curve analysis was performed to assess the ability of the integrated NLR and fibrinogen level to discriminate between NMIBC and MIBC in the validation. The cutoff value of integrated NLR and fibrinogen value was estimated according to the Youden index. With this value, sensitivity was found as $86 \%$, specificity was $42 \%$, and AUC was 0.801 $(P=0.01$, confidence interval $=0.763-0.838)$. Compared to NLR or fibrinogen level alone, we found that the integrated NLR and fibrinogen level had high sensitivity, though the specificity was less. It could help the urologists make better decision on treatment option for patients according to the hematological indexes combined with patient individual preferences and needs.

\section{Limitations}

Some limitations of the present study must be mentioned. First, the information involved in this study were obtained from 669 patients, and the study was conducted in a single center with a small sample; hence, the results cannot be generalized. Including surgeon and pathologist in the study was also a limitation that the proportion of NMIBC and MIBC patients did not correspond to the international epidemiological data. Due to the retrospective nature of this study, the attributable factors suffered from a recall bias and were not analyzable. Second, the role of NLR and fibrinogen level in the cell carcinoma was not investigated in this study.

\section{Conclusion}

In conclusion, immune markers such as NLR and hemostatic factor fibrinogen may assist in distinguishing patients following surgery for a malignant indication. In the current study, we demonstrated that an elevated preoperative NLR and fibrinogen level was associated with long-term progression. Integrated NLR and fibrinogen level may therefore serve as an easily obtained preoperative factor to help determine MIBC and NMIBC of patients following surgery for BCs.

\section{Acknowledgments}

This work was supported by the National Natural Science Foundation of China (grant number 81402095) and Tianjin Municipal Health Bureau Project (No 2014KZ104).

\section{Disclosure}

The authors report no conflicts of interest in this work.

\section{References}

1. Ferlay J, Steliarova-Foucher E, Lortet-Tieulent J, et al. Cancer incidence and mortality patterns in Europe: estimates for 40 countries in 2012. Eur J Cancer. 2013;49(6):1374-1403.

2. DeSantis CE, Lin CC, Mariotto AB, et al. Cancer treatment and survivorship statistics, 2014. CA Cancer J Clin. 2014;64(4):252-271.

3. Tetu B. Diagnosis of urothelial carcinoma from urine. Mod Pathol. 2009;22(Suppl 2):S53-S59.

4. Dal Moro F, Valotto C, Guttilla A, Zattoni F. Urinary markers in the everyday diagnosis of bladder cancer. Urologia. 2013;80(4):265-275.

5. Jakse G, Algaba F, Malmstrom PU, Oosterlinck W. A second-look TUR in T1 transitional cell carcinoma: why? Eur Urol. 2004;45(5):539-546; discussion 46.

6. Stein JP, Lieskovsky G, Cote R, et al. Radical cystectomy in the treatment of invasive bladder cancer: long-term results in 1,054 patients. J Clin Oncol. 2001;19(3):666-675. 
7. Arcangeli G, Strigari L, Arcangeli S. Radical cystectomy versus organ-sparing trimodality treatment in muscle-invasive bladder cancer: a systematic review of clinical trials. Crit Rev Oncol Hematol. 2015;95(3):387-396.

8. van Rhijn BW, van der Poel HG, van der Kwast TH. Urine markers for bladder cancer surveillance: a systematic review. Eur Urol. 2005;47(6): 736-748.

9. Masson-Lecomte A, Rava M, Real FX, Hartmann A, Allory Y, Malats N. Inflammatory biomarkers and bladder cancer prognosis: a systematic review. Eur Urol. 2014;66(6):1078-1091.

10. Zhao QT, Yang Y, Xu S, et al. Prognostic role of neutrophil to lymphocyte ratio in lung cancers: a meta-analysis including 7,054 patients. Onco Targets Ther. 2015;8:2731-2738.

11. Yao M, Liu Y, Jin H, et al. Prognostic value of preoperative inflammatory markers in Chinese patients with breast cancer. Onco Targets Ther. 2014;7:1743-1752.

12. Tang L, Li X, Wang B, et al. Prognostic value of neutrophil-tolymphocyte ratio in localized and advanced prostate cancer: a systematic review and meta-analysis. PLoS One. 2016;11:e0153981.

13. Han S, Liu Y, Li Q, et al. Pre-treatment neutrophil-to-lymphocyte ratio is associated with neutrophil and T-cell infiltration and predicts clinical outcome in patients with glioblastoma. BMC Cancer. 2015;15:617.

14. Galizia G, Lieto E, Zamboli A, et al. Neutrophil to lymphocyte ratio is a strong predictor of tumor recurrence in early colon cancers: a propensity score-matched analysis. Surgery. 2015;158:112-120.

15. Viers BR, Boorjian SA, Frank I, et al. Pretreatment neutrophil-tolymphocyte ratio is associated with advanced pathologic tumor stage and increased cancer-specific mortality among patients with urothelial carcinoma of the bladder undergoing radical cystectomy. Eur Urol. 2014;66:1157-1164.

16. Na N, Yao J, Cheng C, et al. Meta-analysis of the efficacy of the pretreatment neutrophil-to-lymphocyte ratio as a predictor of prognosis in renal carcinoma patients receiving tyrosine kinase inhibitors. Oncotarget. 2016;7(28):44039-44046.

17. Asaoka T, Miyamoto A, Maeda S, et al. Prognostic impact of preoperative NLR and CA19-9 in pancreatic cancer. Pancreatology. 2016;16: 434-440.

18. Degen JL, Palumbo JS. Hemostatic factors, innate immunity and malignancy. Thromb Res. 2012;129(Suppl 1):S1-S5.

19. Degen JL, Palumbo JS. Mechanisms linking hemostatic factors to tumor growth in mice. Pathophysiol Haemost Thromb. 2003;33(Suppl 1): 31-35.

20. Palumbo JS, Degen JL. Hemostatic factors in tumor biology. J Pediatr Hematol Oncol. 2000;22:281-287.

21. Turna H, Ozguroglu M, Bolayirli M, et al. Is there any effect of tumor burden on hemostatic parameters in cancer patients? A case-control study of hemostatic abnormalities and anticardiolipin antibodies in solid tumors. Clin Appl Thromb Hemost. 2009;15:454-457.

22. Raj GV, Karavadia S, Schlomer B, et al. Contemporary use of perioperative cisplatin-based chemotherapy in patients with muscle-invasive bladder cancer. Cancer. 2011;117(2):276-282.

23. Grivennikov SI, Greten FR, Karin M. Immunity, inflammation, and cancer. Cell. 2010;140(6):883-899.

24. Gondo T, Nakashima J, Ohno Y, et al. Prognostic value of neutrophil-tolymphocyte ratio and establishment of novel preoperative risk stratification model in bladder cancer patients treated with radical cystectomy. Urology. 2012;79(5):1085-1091.

25. Azuma T, Matayoshi Y, Odani K, etal. Preoperative neutrophil-lymphocyte ratio as an independent prognostic marker for patients with upper urinary tract urothelial carcinoma. Clin Genitourinary Cancer. 2013;11(3): 337-341.

26. Ozyalvacli ME, Ozyalvacli G, Kocaaslan R, et al. Neutrophil-lymphocyte ratio as a predictor of recurrence and progression in patients with high-grade pT1 bladder cancer. Can Urol Assoc J. 2015;9(3-4):E126-E131.
27. Ceylan C, Doluoglu OG, Keles I, et al. Importance of the neutrophil-tolymphocyte ratio in muscle-invasive and non-muscle invasive bladder tumors. Urologia. 2014;81(2):120-124.

28. Potretzke A, Hillman L, Wong K, et al. NLR is predictive of upstaging at the time of radical cystectomy for patients with urothelial carcinoma of the bladder. Urol Oncol. 2014;32(5):631-636.

29. Paramanathan A, Saxena A, Morris DL. A systematic review and metaanalysis on the impact of pre-operative neutrophil lymphocyte ratio on long term outcomes after curative intent resection of solid tumours. Surg Oncol. 2014;23(1):31-39.

30. Templeton AJ, McNamara MG, Seruga B, et al. Prognostic role of neutrophil-to-lymphocyte ratio in solid tumors: a systematic review and meta-analysis. J Natl Cancer Inst. 2014;106(6):dju124.

31. Demirtas A, Sabur V, Akinsal EC, et al. Can neutrophil-lymphocyte ratio and lymph node density be used as prognostic factors in patients undergoing radical cystectomy? ScientificWorldJournal. 2013; 2013:703579.

32. Luo HL, Chen YT, Chuang YC, et al. Subclassification of upper urinary tract urothelial carcinoma by the neutrophil-to-lymphocyte ratio (NLR) improves prediction of oncological outcome. BJU Int. 2014;113(5b): E144-E149.

33. Wei Y, Jiang YZ, Qian WH. Prognostic role of NLR in urinary cancers: a meta-analysis. PLoS One. 2014;9(3):e92079.

34. Polterauer S, Grimm C, Seebacher V, et al. Plasma fibrinogen levels and prognosis in patients with ovarian cancer: a multicenter study. Oncologist. 2009;14(10):979-985.

35. Krenn-Pilko S, Langsenlehner U, Stojakovic T, et al. An elevated preoperative plasma fibrinogen level is associated with poor disease-specific and overall survival in breast cancer patients. Breast. 2015;24(5): 667-672.

36. Pichler M, Hutterer GC, Stojakovic T, Mannweiler S, Pummer K, Zigeuner R. High plasma fibrinogen level represents an independent negative prognostic factor regarding cancer-specific, metastasis-free, as well as overall survival in a European cohort of non-metastatic renal cell carcinoma patients. Br J Cancer. 2013;109(5):1123-1129.

37. Zhao K, Deng H, Qin Y, Liao W, Liang W. Prognostic significance of pretreatment plasma fibrinogen and platelet levels in patients with earlystage cervical cancer. Gynecol Obstet Invest. 2015;79(1):25-33.

38. Seebacher V, Polterauer S, Grimm C, et al. The prognostic value of plasma fibrinogen levels in patients with endometrial cancer: a multicentre trial. Br J Cancer. 2010;102(6):952-956.

39. Wayman J, O’Hanlon D, Hayes N, Shaw I, Griffin SM. Fibrinogen levels correlate with stage of disease in patients with oesophageal cancer. Br J Surg. 1997;84(2):185-188.

40. Tang L, Liu K, Wang J, Wang C, Zhao P, Liu J. High preoperative plasma fibrinogen levels are associated with distant metastases and impaired prognosis after curative resection in patients with colorectal cancer. J Surg Oncol. 2010;102(5):428-432.

41. Thurner EM, Krenn-Pilko S, Langsenlehner U, et al. The association of an elevated plasma fibrinogen level with cancer-specific and overall survival in prostate cancer patients. World J Urol. 2015;33(10): 1467-1473.

42. Ghezzi F, Cromi A, Siesto G, et al. Prognostic significance of preoperative plasma fibrinogen in endometrial cancer. Gynecol Oncol. 2010; 119(2):309-313.

43. Jiang HG, Li J, Shi SB, et al. Value of fibrinogen and D-dimer in predicting recurrence and metastasis after radical surgery for non-small cell lung cancer. Med Oncol. 2014;31(7):22.

44. Lee JH, Hyun JH, Kim DY, et al. The role of fibrinogen as a predictor in preoperative chemoradiation for rectal cancer. Ann Surg Oncol. 2015;22(1):209-215. 


\section{Publish your work in this journal}

OncoTargets and Therapy is an international, peer-reviewed, open access journal focusing on the pathological basis of all cancers, potential targets for therapy and treatment protocols employed to improve the management of cancer patients. The journal also focuses on the impact of management programs and new therapeutic agents and protocols on

patient perspectives such as quality of life, adherence and satisfaction. The manuscript management system is completely online and includes a very quick and fair peer-review system, which is all easy to use. Visit http://www.dovepress.com/testimonials.php to read real quotes from published authors.

Submit your manuscript here: http://www.dovepress.com/oncotargets-and-therapy-journal 